JOURNAL OF

SYMPLECTIC GEOMETRY

Volume 4, Number 1, 63-70, 2006

\title{
SYMPLECTIC 4-MANIFOLDS WITH ARBITRARY FUNDAMENTAL GROUP NEAR THE BOGOMOLOV-MIYAOKA-YAU LINE
}

\author{
Scott BALDRIDGe AND PAUl KirK
}

In this paper, we construct a family of symplectic 4-manifolds with positive signature for any given fundamental group $G$ that approaches the BMY line. The family is used to show that one cannot hope to do better than the BMY inequality in finding a lower bound for the function $f=\chi+b \sigma$ on the class of all minimal symplectic 4-manifolds with a given fundamental group.

\section{Introduction}

Let $\chi(S)$ and $\sigma(S)$ denote the Euler characteristic and signature of a closed 4-manifold, respectively. Minimal complex surfaces $S$ of general type satisfy $c_{1}^{2}(S)>0, \chi(S)>0$ and

$$
2 \chi_{h}(S)-6 \leq c_{1}^{2}(S) \leq 9 \chi_{h}(S),
$$

where $c_{1}^{2}(S)=2 \chi(S)+3 \sigma(S)$ and $\chi_{h}(S)=\frac{1}{4}(\chi(S)+\sigma(S))$. The second inequality is usually referred to as the Bogomolov-Miyaoka-Yau inequality. Finding symplectic (or Kähler) 4-manifolds on or near the BMY line has a long and interesting history $[\mathbf{2}, \mathbf{3}, \mathbf{5}, \mathbf{8}-\mathbf{1 1}]$.

All known examples of symplectic 4-manifolds on the BMY line, except $\mathbb{C P}^{2}$, have large fundamental groups. In fact, if $S$ is a complex surface differing from $\mathbb{C P}^{2}$, the equality $c_{1}^{2}(S)=9 \chi_{h}(S)$ holds if and only if the unit disk $D^{4}=\left\{\left.\left(z_{1}, z_{2}\right) \in \mathbb{C}^{2}|| z_{1}\right|^{2}+\left|z_{2}\right|^{2} \leq 1\right\}$ covers $S[\mathbf{6}, \mathbf{7}, \mathbf{1 3}]$ and hence $\left|\pi_{1}(S)\right|=\infty$. One goal of 4-dimensional symplectic topology is to produce examples that fill in the geography with respect to $\left(c_{1}^{2}, \chi_{h}\right)$. In this article, we are interested in what can be said for a given fundamental group.

Stipsicz $[\mathbf{1 1}]$ constructed simply connected symplectic 4-manifolds $C_{n}$ for which $c_{1}^{2}\left(C_{n}\right) / \chi_{h}\left(C_{n}\right) \rightarrow 9$ as $n \rightarrow \infty$. Our main theorem generalizes this result to any fundamental group. 
Theorem 1.1. Let $G$ have a presentation with g generators $x_{1}, \ldots, x_{g}$ and $r$ relations $w_{1}, \ldots, w_{r}$. For each integer $n>1$, there exists a symplectic 4-manifold $M(G, n)$ with fundamental group $G$ with Euler characteristic

$$
\chi(M(G, n))=75 n^{2}+256 n+130+12(g+r+1),
$$

and signature

$$
\sigma(M(G, n))=25 n^{2}-68 n-78-8(g+r+1) .
$$

Our interest in this question developed while investigating pairs $(a, b) \in$ $\mathbb{R}^{2}$ for which the function $f=a \chi+b \sigma$ has a lower bound on the class of symplectic manifolds with a given fundamental group [1]. In that article, we considered the following.

Fix a finitely presented group $G$ and let $\mathfrak{M}$ denote either the class $\mathfrak{M}(G)$ of closed symplectic 4-manifolds with fundamental group $G$ or the class $\mathfrak{M}^{\min }(G)$ of minimal, closed symplectic 4-manifolds with fundamental group $G$.

For $b \in \mathbb{R}$, define $f_{\mathfrak{M}}(b) \in \mathbb{R} \cup\{-\infty\}$ to be the infimum

$$
f_{\mathfrak{M}}(b)=\inf _{M \in \mathfrak{M}}\{\chi(M)+b \sigma(M)\}
$$

(In $[\mathbf{1}]$, we considered the infimum $f_{\mathfrak{M}}(a, b)$ of $a \chi+b \sigma$ on $\mathfrak{M}$ and showed that if $a \leq 0$, the infimum is $-\infty$. Thus we restrict to $f_{\mathfrak{M}}(1, b)$, which we more compactly denote by $f_{\mathfrak{M}}(b)$ in the present article.)

We showed in [1] that the set

$$
D_{\mathfrak{M}}=\left\{b \mid f_{\mathfrak{M}}(b) \neq-\infty\right\}
$$

(the domain of $f_{\mathfrak{M}}$ ) is an interval satisfying

$$
[-1,1] \subset D_{\mathfrak{M}(G)} \subset(-\infty, 1] \text { and }\left[-1, \frac{3}{2}\right] \subset D_{\mathfrak{M}^{\min }(G)} \subset\left(-\infty, \frac{3}{2}\right] .
$$

The upper bounds are sharp; in fact $1 \in D_{\mathfrak{M}(G)}$ and $\frac{3}{2} \in D_{\mathfrak{M}^{\min }(G)}$.

We are interested in the value of the left endpoint $e_{G}$ of $D_{\mathfrak{M}(G)}$, which is an intriguing invariant of a group $G$. (It may or may not be contained in $D_{\mathfrak{M}(G)}$.) Since $e_{G} \leq-1$, a straightforward argument shows that $e_{G}$ is also the left endpoint of $D_{\mathfrak{M}^{\min }(G)}$.

In [1], we observed that the results of Stipsicz gives a better lower bound (than $-\infty$ ) when $G$ is the trivial group, and so a consequence of the result of this article is an extension to all $G$. In fact Theorem 1.1 easily implies the following corollary.

Corollary 1.2. For any finitely presented group $G$,

$$
D_{\mathfrak{M}(G)} \subset[-3,1] \text { and } D_{\mathfrak{M}^{\min }(G)} \subset\left[-3, \frac{3}{2}\right] .
$$


The BMY inequality $c_{1}^{2} \leq 9 \chi_{h}$ is equivalent to $f_{\mathfrak{M}^{\min }(G)}(-3) \geq 0$ provided $G$ is not a surface group. Hence, the BMY conjecture and Corollary 1.2 together imply that $e_{G}=-3$. Thus, a weaker form of the BMY conjecture could be stated as follows.

Conjecture 1.3 (Weak BMY Conjecture). For each finitely presented group $G, e_{G}=-3$.

\section{The construction}

We use the following notation. If $X$ and $Y$ are symplectic 4-manifolds containing closed genus $g$ symplectic surfaces $F_{X} \subset X$ and $F_{Y} \subset Y$ such that $F_{X}^{2}+F_{Y}^{2}=0$, then the symplectic sum [4] of $X$ and $Y$ along $F_{X}$ and $F_{Y}$ will be denoted by

$$
X \#_{F_{X}, F_{Y}} Y \text {. }
$$

Recall that topologically $X \#_{F_{X}, F_{Y}} Y$ is obtained by removing tubular neighborhoods of $F_{X}$ and $F_{Y}$ and identifying the resulting boundaries, which are $S^{1}$ bundles over a genus $g$ surface, by a fiber-preserving, orientation reversing diffeomorphism.

The symplectic sum admits a symplectic structure so that any symplectic surface in $X-F_{X}$ or $Y-F_{Y}$ remains symplectic in $X \#_{F_{X}, F_{Y}} Y$. Moreover, if $E_{X} \subset X$ (resp. $E_{Y} \subset Y$ ) is a symplectic surface intersecting $F_{X}$ once transversally (resp. intersecting $F_{Y}$ transversally), then the symplectic sum can be constructed so that (the connected sum) $E_{X} \# E_{Y}$ is a symplectic surface in $X \#_{F_{X}, F_{Y}} Y$.

2.1. The first piece: symplectic manifolds with given fundamental group. The following theorem was proven in $[\mathbf{1}]$.

Theorem 2.1. Let $G$ have a presentation with g generators $x_{1}, \ldots, x_{g}$ and $r$ relations $w_{1}, \ldots, w_{r}$. Then there exists a symplectic 4-manifold $M(G)$ with $\pi_{1}(M(G)) \cong G$, Euler characteristic $\chi(M(G))=12(g+r+1)$, and signature $\sigma(M(G))=-8(g+r+1)$.

We will use the following fact. The manifold $M(G)$ constructed in Theorem 2.1 is obtained by taking symplectic sums of a certain base manifold with $g+r+1$ copies of the basic elliptic surface $E(1)$. Since $E(1)$ admits a singular fibration with symplectic generic fibers and six cusp fibers (which are simply connected), so does $E(1)-F$, where $F$ denotes the generic fiber in $E(1)$ along which the symplectic sum giving $M$ is constructed. Thus each $M(G)$ contains a symplectic torus $T_{0}$ such that the induced homomorphism $\pi_{1}\left(T_{0}\right) \rightarrow \pi_{1}(M(G))$ is trivial.

2.2. The second piece: symplectic manifolds near the BMY line. In [11], Stipsicz proved the following theorem. 
Proposition 2.2 (Stipsicz). For each non-negative integer $n$, there exists a symplectic 4-manifold $X(n)$ which admits a genus- $(15 n+1)$ Lefschetz fibration with a section $T_{n+2}$ of genus $(n+2)$ and self-intersection $-(n+1)$. Furthermore, $X(n)$ can be equipped with a symplectic structure such that $T_{n+2}$ is a symplectic submanifold. The projection map $X(n) \rightarrow T_{n+2}$ induces an isomorphism on fundamental groups. The Euler characteristic of $X(n)$ is $\chi(X(n))=75 n^{2}+180 n+12$ and the signature is $\sigma(X(n))=25 n^{2}-60 n-8$.

Denote by $F_{15 n+1} \subset X(n)$ a fixed generic fiber of $X(n)$. This is a symplectic surface with trivial normal bundle.

2.3. The third piece: a simply connected manifold. Gompf constructs a symplectic 4-manifold $S_{1,1}$ in [4, Lemma 5.5] which contains a disjoint pair $T, F$ of symplectically embedded surfaces $T$ of genus one and $F$ of genus two, with trivial normal bundles such that $S_{1,1}-(T \cup F)$ is simply connected. Thus, the symplectic sum $A$ of two copies $S_{1,1}$ along the genus two surfaces

$$
A=S_{1,1} \#_{F, F} S_{1,1}
$$

contains a pair of disjointly embedded symplectic tori $T_{1} \cup T_{2} \subset A$ with trivial normal bundles so that the complement $A-\left(T_{1} \cup T_{2}\right)$ is simply connected. Since $S_{1,1}$ has Euler characteristic 23 and signature $-15, \chi(A)=50$ and $\sigma(A)=-30$.

The manifold $A$ has a useful property, whose proof is a simple application of the Seifert-Van Kampen theorem.

Proposition 2.3. Suppose $B$ and $C$ are symplectic 4-manifolds containing symplectic tori $i_{B}: T_{B} \subset B$ and $i_{C}: T_{C} \subset C$ with trivial normal bundles.

Let $D=B \#_{T_{B}, T_{1}} A \#_{T_{2}, T_{C}} C$ be the symplectic sum of $B, A$, and $C$. Then

$$
\pi_{1}(D)=\left(\frac{\pi_{1}(B)}{N\left(\left(i_{B}\right)_{*}\left(\pi_{1}\left(T_{B}\right)\right)\right.}\right) \star\left(\frac{\pi_{1}(C)}{N\left(\left(i_{C}\right)_{*}\left(\pi_{1}\left(T_{C}\right)\right)\right.}\right)
$$

where $\star$ denotes free product and $N(H)$ denotes the normal closure of a subgroup $H$.

2.4. The fourth piece: an elbow. Let $T$ be a torus and $\{a, b\}$ a pair of smoothly embedded loops forming a symplectic basis of $\pi_{1} T$. Let $\varphi: T \rightarrow T$ be the Dehn twist around $a$. The mapping torus $Y_{\phi}$ fibers over $S^{1}$ with fiber $T$. Let $t_{1}: S^{1} \rightarrow Y_{\phi}$ denote a section. Taking a product of $Y_{\phi}$ with $S^{1}$ yields a symplectic 4-manifold $Y_{\phi} \times S^{1}$ (this is just Thurston's manifold from [12]) which fibers over a torus with symplectic torus fibers. Moreover, the symplectic structure can be chosen so that the section $t_{1} \times$ id $: S^{1} \times S^{1} \rightarrow$ $Y_{\phi} \times S^{1}$ is symplectic. Denote by $s_{1}: S^{1} \rightarrow\{p\} \times S^{1} \subset Y_{\phi} \times S^{1}$ the loop representing the second factor.

Note that $Y_{\phi} \times S^{1}$ contains a torus $T^{\prime}=b \times s_{1}$, where $b$ is the curve described above in the fiber of $Y_{\phi}$. The torus $T^{\prime}$ is homologically non-trivial 
by the Kunneth theorem, since $b$ is non-trivial in $H_{1}\left(Y_{\phi}\right)$. Moreover, $T^{\prime}$ is Lagrangian with respect to the symplectic structure on $Y_{\phi} \times S^{1}$. Thus, the symplectic structure on $Y_{\phi} \times S^{1}$ can be perturbed slightly to make $T^{\prime}$ symplectic by adding a small closed 2-form that restricts to a volume form on $T^{\prime}$. Note moreover that $T^{\prime}$ is disjoint from the section $t_{1} \times s_{1}: S^{1} \times S^{1} \rightarrow$ $Y_{\phi} \times S^{1}$ since we can assume that $t_{1}$ intersects the fiber containing $b$ in a point which does not lie on $b$. The tubular neighborhood of $T^{\prime}$ in $Y_{\phi} \times S^{1}$ is trivial since $b$ can isotoped off itself in a fiber of $Y_{\phi} \rightarrow S^{1}$. Similarly the tubular neighborhood of the section $t_{1} \times s_{1}$ is trivial since $t_{1}$ can be pushed off itself in $Y_{\phi}$.

Define $\operatorname{Elb}(n)$ to be the symplectic sum $\operatorname{Elb}(n)=\left(Y_{\phi} \times S^{1}\right) \#_{T, T^{2}}\left(T^{2} \times\right.$ $\left.\Sigma_{n-1}\right)$. The symplectic sum can be carried out so that the sections of $Y_{\phi} \times$ $S^{1} \rightarrow S^{1} \times S^{1}$ and $T^{2} \times \Sigma_{n-1} \rightarrow \Sigma_{n-1}$ yield a symplectic section of the resulting fibration $\operatorname{Elb}(n) \rightarrow \Sigma_{n}$. Thus, $\operatorname{Elb}(n)$ contains a disjoint pair of symplectic surfaces with trivial normal bundles, a torus $T^{\prime}=b \times s_{1}$, and a genus $n$ surface, the image of the section, which we denote by $D_{n}$.

Letting $t_{2}, s_{2}, \ldots, t_{n}, s_{n}$ denote the generators of $\pi_{1}\left(\Sigma_{n-1}\right)$, one computes

$$
\begin{aligned}
\pi_{1}(\operatorname{Elb}(n))= & \left\langle a, b, t_{1}, s_{1}, \ldots, t_{n}, s_{n}\right| a \text { central, }\left[b, t_{1}\right]=a, \\
& {\left[b, t_{i}\right]=1 \text { for } i>1,\left[b, s_{i}\right]=1 \text { for all } i, } \\
& \left.\prod_{i=1}^{n}\left[t_{i}, s_{i}\right]=1\right\rangle .
\end{aligned}
$$

The inclusion of $T^{\prime}$ into $\operatorname{Elb}(n)$ takes the generators of $\pi_{1} T^{\prime}$ to $b$ and $s_{1}$, and the inclusion of $D_{n}$ takes the standard surface group generators to $t_{1}, s_{1}, \ldots, t_{n}, s_{n}$. The Euler characteristic and signature of $\operatorname{Elb}(n)$ both vanish.

The manifold $\operatorname{Elb}(n)-D_{n}$ is a punctured torus fibration over $\Sigma_{n}$ and hence has a presentation with the same generators and all the same relations except that one no longer has $a$ commuting with $b$, i.e., $a$ commutes with all generators except $b$.

2.5. The fifth piece: an elliptic surface. We find a symplectically embedded surface $J$ of genus $n+3$ and self-intersection $n+1$ in the elliptic surface $E(n+5)$ such that $E(n+5)-J$ is simply connected as follows. Consider $n+3$ copies of the generic fiber and one copy of the section in a fibration $E(n+5) \rightarrow \mathbb{C P}^{1}$ with $6(n+5)$ cusp fibers. The section and fibers are symplectic with regards to the symplectic structure on the elliptic fibration $E(n+5)$. Resolve the $n+3$ transverse double points [4] to get a symplectically embedded surface $J$ of genus $n+3$ and self-intersection $n+1$ (the fiber hits the section once and that section has self-intersection $-(n+5))$. The complement $E(n+5)-J$ is simply connected because $E(n+5)$ has a simply connected fiber which intersects $J$ in one point: the normal circle of a tubular neighborhood of $J$ is nullhomotopic in $E(n+5)-J$.

2.6. Putting the pieces together. We begin by a modification of Stipsicz's construction. Let $Z(n)$ be the symplectic sum of $\operatorname{Elb}(15 n+1)$ 
and $X(n)$ along $D_{15 n+1} \subset \operatorname{Elb}(15 n+1)$ and the fiber $F_{15 n+1}$ of the Lefschetz fibration $X(n) \rightarrow \Sigma_{n+2}$

$$
Z(n)=\operatorname{Elb}(15 n+1) \#_{D_{15 n+1}, F_{15 n+1}} X(n) .
$$

The symplectic sum can be constructed so that the fiber $T \subset \operatorname{Elb}(15 n+1)$ and the section $T_{n+2} \subset X(n)$ add to yield a symplectic surface of genus $n+3, K_{n+3}=T \# T_{n+2} \subset Z(n)[4]$. The important property of $Z(n)$ is that it contains a symplectic torus $T^{\prime}$, since $D_{15 n+1}$ and $T^{\prime}$ are disjoint.

The fundamental group of $Z(n)$ is easily computed, since Elb $(15 n+1)-$ $D_{15 n+1}$ is a fiber bundle with punctured torus fibers and $X(n)-F_{15 n+1}$ is a Lefschetz fibration over a punctured genus $n+2$ surface with at least one simply connected fiber. Using the Seifert-Van Kampen theorem and Novikov additivity one obtains the following.

Lemma 2.4. The fundamental group of $Z(n)$ is the free product of $\mathbb{Z}$ with generator $b$ and a genus $n+2$ surface group generated by $x_{i}, y_{i}$ :

$$
\pi_{1}(Z(n))=\mathbb{Z} b \star\left\langle x_{i}, y_{i}, i=1, \ldots, n+2 \mid \prod\left[x_{i}, y_{i}\right]=1\right\rangle .
$$

The symplectic manifold $Z(n)$ contains a disjoint pair of symplectic surfaces, $T^{\prime} \cup K_{n+3} \subset Z(n)$ satisfying $\left[T^{\prime}\right]^{2}=0$, and $\left[K_{n+3}\right]^{2}=-n-1$. The induced homomorphism $\pi_{1}\left(T^{\prime}\right) \rightarrow \pi_{1}(Z(n))$ is the map

$$
\left\langle a, s_{1} \mid\left[a, s_{1}\right]\right\rangle \longrightarrow \pi_{1} Z(n) \quad a \longmapsto a, s_{1} \longmapsto 1 .
$$

The induced homomorphism $\pi_{1}\left(K_{n+3}\right) \rightarrow \pi_{1}(Z(n))$ is the map

$$
\begin{gathered}
\left\langle a, b, x_{1}, y_{1}, \ldots, x_{n+2}, y_{n+2} \mid[a, b] \prod\left[x_{i}, y_{i}\right]=1\right\rangle \longrightarrow \pi_{1}(Z(n)) \\
a \longmapsto a, \quad b \longmapsto 1, \quad x_{i} \longmapsto x_{i}, \quad y_{i} \longmapsto y_{i} .
\end{gathered}
$$

Moreover, $\chi(Z(n))=75 n^{2}+240 n+12$ and $\sigma(Z(n))=25 n^{2}-60 n-8$.

The symplectic sum of $Z(n)$ with $E(n+5)$ along $J, Z(n) \#_{K_{n+3}, J} E(n+5)$ is a simply connected symplectic 4 -manifold containing a torus $T_{1}$ with trivial normal bundle and appropriate Euler characteristic and signature. We take symplectic sum of this manifold with $A$ to obtain an example with a torus whose complement is simply connected.

Define $W(n)$ to be the symplectic sum

$$
W(n)=A \#_{T_{1}, T^{\prime}} Z(n) \#_{K_{n+3}, J} E(n+5) .
$$

Then since $\pi_{1}\left(A-\left(T_{2} \cup T_{2}\right)\right)=1$, the following proposition follows straightforwardly.

Proposition 2.5. The symplectic manifold $W(n)$ is simply connected and contains a symplectic torus $T_{2} \subset W(n)$ with trivial normal bundle so that 
$\pi_{1}\left(W(n)-T_{2}\right)=1$. It has Euler characteristic $\chi(W(n))=75 n^{2}+256 n+130$ and signature $\sigma(W(n))=25 n^{2}-68 n-78$.

We can now prove Theorem 1.1.

Proof of Theorem 1.1. The symplectic sum

$$
M(G, n)=M(G) \#_{T_{0}, T_{2}} W(n)
$$

has fundamental group $G$ by Proposition 2.3. The calculations of $\chi(M(G, n))$ and $\sigma(M(G, n))$ are routine.

\section{References}

[1] S. Baldridge and P. Kirk, On symplectic 4-manifolds with prescribed fundamental group, Commentarii Math. Helv. E-print GT0504345, in press.

[2] Z. Chen, On the geography of surfaces - simply connected minimal surfaces with positive index, Math. Ann. 126 (1987), 141-164.

[3] Z. Chen, The existence of algebraic surfaces with preassigned Chern numbers, Math. Z. 206 (1991), 241-254.

[4] R. Gompf, A new construction of symplectic manifolds, Ann. Math. 142 (1995), $527-595$.

[5] R. Gompf and A. Stipsicz, 4-Manifolds and Kirby calculus, in 'Graduate studies in mathematics,' 20, American Mathematical Society, Providence, Rhode Island, 1999.

[6] F. Hirzebruch, The signature of ramified coverings, in 'Global Analysis' (Papers in Honor of K. Kodaira), (D. Spencer and S. Iyanaga, eds.), Princeton University Press, 1969, 253-265.

[7] Y. Miyaoka, The maximal number of quotient singularities on surfaces with given numerical invariants, Math. Ann. 268 (1984), 159-171.

[8] B. Moishezon and M. Teicher, Simply connected algebraic surfaces of positive index, Invent. Math. 89 (1987), 601-644.

[9] U. Persson, C. Peters and G. Xiao, Geography of spin surfaces, Topology 35 (1996), 845-862.

[10] A. Stipsicz, Simply connected 4-manifolds near the Bogomolov-Miyaoka-Yau line, Math. Res. Lett. 5 (1998), 723-730.

[11] A. Stipsicz, Simply connected symplectic 4-manifolds with positive signature, Turk. J. Math. 23 (1999), 145-150, Proceedings of 6th Gökova Geometry-Topology Conference.

[12] W.P. Thurston, Some simple examples of symplectic manifolds, Proc. Amer. Math. Soc. 55(2) (1976), 467-468.

[13] S.-T. Yau, Calabi's conjecture and some new results in algebraic geometry, Proc. Nat. Acad. Sci. USA 74 (1977), 1798-1799.

Department of Mathematics

LOUISIANA STATE UNIVERSITY

BATON Rouge

LA 70803

E-mail address: sbaldrid@math.lsu.edu 
Mathematics Department

INDIANA UNIVERSITY

BLOOMINGTON

IN 47405

E-mail address: pkirk@indiana.edu

Received 07/28/2005, accepted 12/02/2005.

The first author gratefully acknowledges support from the NSF grant DMS-0507857. The second author gratefully acknowledges support from the NSF grant DMS-0202148. 